\title{
Sensing electromagnetic fields with the AC-Stark effect in two-photon spectroscopy of cold trapped $\mathrm{HD}^{+}$
}

\author{
F. L. Constantin*a \\ ${ }^{a}$ Laboratoire PhLAM, CNRS UMR 8523, 59655 Villeneuve d'Ascq, France
}

\begin{abstract}
This contribution evaluates the potential for SI-traceable measurements of electromagnetic fields from precision measurements of two-photon rovibrational transitions of cold trapped $\mathrm{HD}^{+}$ions interpreted with accurate theoretical models. Zeeman spectroscopy of a hyperfine component of the $(\mathrm{v}, \mathrm{L})=(0,0) \rightarrow(2,2)$ two-photon transition is exploited for the measurement of a static magnetic field. The absolute sensitivity and accuracy is estimated at the $10^{-10} \mathrm{~T}$ level in the case of frequency measurements at the quantum projection noise limit. Measurements of the AC-Stark shifts of different Zeeman components of the $(\mathrm{v}, \mathrm{L})=(0,0) \rightarrow(2,0)$ two-photon transition at different orientations of the magnetic field are exploited to measure the polarisation and the intensity of a THz-wave off-resonantly coupled to $\mathrm{HD}^{+}$rotational levels. The sensitivity is estimated at the $10^{-7} \mathrm{~W} / \mathrm{m}^{2}$ level. A reference THz-wave with an intensity of $1 \mathrm{~W} / \mathrm{m}^{2}$ can be calibrated in intensity with a fractional accuracy limited at the $10^{-2}$ level by the accuracy of the theoretical calculations and at the $10^{-4}$ level by the experimental errors. In addition, an approach for retrieval of the full polarisation ellipse is demonstrated with a selected $\mathrm{THz}$-wave. The fractional accuracy of the calibration is better than $5 \%$ for the amplitudes and better than $10 \%$ for the phases of the electric field components of the THz-wave.
\end{abstract}

Keywords: cold trapped ions, hydrogen molecular ions, electrometry, two-photon spectroscopy, ac-Stark shift, dynamic polarizability, polarization ellipse, reference frames

\section{INTRODUCTION}

Precise sensing of electromagnetic fields has applications in inertial navigation, definition of frequency standards, geophysical exploration and biological imaging. Particularly, the detection of microwave fields by atomic spectroscopy may provide SI-traceable measurements, sub-wavelength resolution, accuracy, precision and long-term reproducible operation $^{1}$, comparing to the traditional technology with an antenna and a solid-state rectifier. The Rydberg states are highly sensitive to microwave electric fields. Rabi oscillations in Rydberg atom spectroscopy allowed building compact electric field sensors in vapour cells ${ }^{2}$. The hydrogen molecular ions are simple quantum systems for which transition frequencies $^{3}$ and systematic shifts ${ }^{4,5}$ were calculated accurately. The experiments exploited co-trapping with laser-cooled $\mathrm{Be}^{+}$ions in Paul traps for sympathetical cooling and spatial confinement for $\mathrm{HD}^{+}$. Spectra of $\mathrm{HD}^{+}$were efficiently detected by dissociation and a mass-spectroscopy technique. A relatively small static magnetic field was used to indicate the quantification axis and to split $\mathrm{HD}^{+}$magnetic levels. The rovibrational lines were measured with $10^{-9}$ precision by Doppler-limited spectroscopy ${ }^{6}$. Doppler-free rotational spectroscopy in the Lamb-Dicke regime of cold trapped $\mathrm{HD}^{+}$ions provided $10^{-10}$-level precision for the $(\mathrm{v}, \mathrm{L})=(0,0) \rightarrow(0,1)$ transition at $1.3 \mathrm{THz}^{7}$.

Two-photon infrared spectroscopy, addressed in this contribution, may provide resolution and accuracy at the $10^{-12}$-level or better ${ }^{8}$. This contribution evaluates the performances of Zeeman spectroscopy to measure the magnitude of a static magnetic field. A new approach for THz-wave electrometry is proposed, based on measurements of the AC-Stark shifts induced on Zeeman sub-components of a two-photon rovibrational transition of $\mathrm{HD}^{+}$.

\section{TWO-PHOTON SPECTROSCOPY OF HD ${ }^{+}$}

The hydrogen molecular ions, as the simplest molecules, benefit from accurate models for their energy levels. The main contribution to the energy is the rovibrational energy, calculated neglecting the hyperfine structure and in absence of perturbation from external fields. The ab-initio results are expressed as the sum of the nonrelativistic Scrödinger energy with a series expansion of corrections taking into account the relativistic, radiative and nuclear-size related contributions

*fl.constantin@univ-lille1.fr 
(the most accurate calculations were reported in ref. ${ }^{3}$ ). The next important contribution comes from the hyperfine energy, which was calculated in the nonrelativistic limit at the order $\alpha^{2}$ using a Breit-Pauli spin Hamiltonian ${ }^{9}$ and the basis vectors $|\mathrm{n}>=|$ vLFSJ $>$ from the coupling $\overrightarrow{\mathrm{F}}=\overrightarrow{\mathrm{S}}_{\mathrm{e}}+\overrightarrow{\mathrm{I}}_{\mathrm{p}} ; \overrightarrow{\mathrm{S}}=\overrightarrow{\mathrm{F}}+\overrightarrow{\mathrm{I}}_{\mathrm{d}} ; \overrightarrow{\mathrm{J}}=\overrightarrow{\mathrm{L}}+\overrightarrow{\mathrm{S}}$ of the rotational angular momentum $\overrightarrow{\mathrm{L}}$

with the spins of the proton $\vec{I}_{p}$, deuteron $\vec{I}_{d}$, electron $\vec{S}_{e}$, to yield the total angular momentum $\vec{J}$. In presence of a small magnetic field, the hyperfine levels are split into magnetic sublevels labelled with the quantum number $J_{z}$ of the projection of $\overrightarrow{\mathrm{J}}$ on the magnetic field axis. The total energy of a Zeeman level is expressed as:

$$
\begin{aligned}
& E\left(v, L, F, S, J, J_{z}\right)=E_{r v}(v, L)+E_{h f}(v, L, F, S, J)+\Delta E_{z}\left(v, L, F, S, J, J_{z} ; B\right) \\
& E\left(v, L, F, S, J, J_{z} ; B\right)-E\left(v, L, F, S, J, J_{z} ; B=0\right) \approx h\left[t_{v, L, F, S, J, J_{z}} J_{z} B+\left(q_{v, L, F, S, J, J}+r_{v, L, F, S, J, J_{z}} J_{z}^{2}\right) B^{2}\right]
\end{aligned}
$$

In the previous equation, the Zeeman energy sublevels $\left|n, J_{Z}\right\rangle$ are approximated with a quadratic dependence depending on parameters $t, q$, and $r$ that have been calculated in ref. ${ }^{4}(h$ is the Planck constant). Figure 1 presents a selection of Zeeman energy levels that are addressed by two-photon spectroscopy and some off-resonant couplings that are exploited for THz-wave sensing.

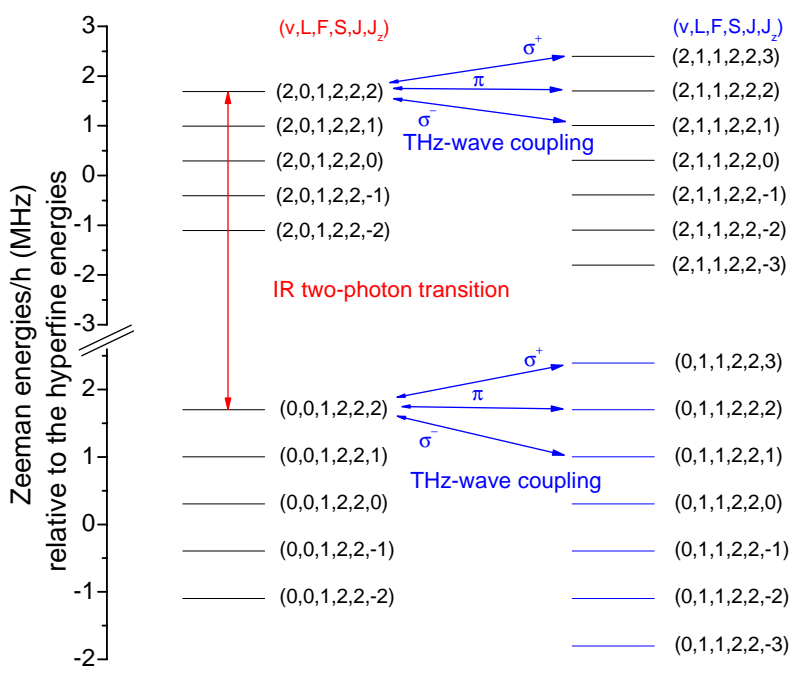

Figure 1. Zeeman components of the hyperfine energy levels of $\mathrm{HD}^{+}$ions addressed in two-photon spectroscopy and relevant couplings for THz-wave sensing.

The proposed experimental setup is sketched in Figure 2. About $10^{2} \mathrm{HD}^{+}$ions are co-trapped with $\sim 10^{3}$ laser-cooled $\mathrm{Be}^{+}$ ions to form a Coulomb crystal in a radiofrequency trap. The detection of the rovibrational transitions is performed by REMPD $^{10}$. The fluorescence at $313 \mathrm{~nm}$ from the laser-cooled Be ${ }^{+}$ions is used to monitor the loss of trapped $\mathrm{HD}^{+}$ions when the secular motion of $\mathrm{HD}^{+}$ions is excited in the trap. A static magnetic field, controlled with three orthogonal coil pairs, splits the two-photon rovibrational transitions in $\sigma^{ \pm}\left(\Delta \mathrm{J}_{\mathrm{z}}= \pm 2\right)$ and $\pi\left(\Delta \mathrm{J}_{\mathrm{z}}=0\right)$ components. The orientation of the magnetic field defines the quantisation axis, by assuming that the THz magnetic field is negligible as well as the effects (change in populations, mixing of energy levels) of the coupling of the THz electric field to the $\mathrm{HD}^{+}$energy levels. This contribution investigates the components of the two-photon rovibrational transition $(\mathrm{v}, \mathrm{L})=(0,0) \rightarrow(2,0) \mathrm{of} \mathrm{HD}^{+}$. Dopplerfree spectroscopy is performed with two counterpropagating lasers tuned around the rovibrational transition at 55.909 THz. The population transferred to the $(\mathrm{v}, \mathrm{L})=(2,0)$ level is dissociated with a $175 \mathrm{~nm}$ laser. The interaction with blackbody radiation at room temperature recycles continuously population in the ground vibrational level of $\mathrm{HD}^{+}$. The change of the population in the rovibrational levels, upon application of the spectroscopy and dissociation lasers, was calculated with a set of rate equations in order to derive the lineshape of the two-photon resonance ${ }^{8}$. The full-width half 
measured linewidth is $20 \mathrm{~Hz}$, for a two-photon transition rate of $10 \mathrm{~s}^{-1}$, a dissociation rate of $200 \mathrm{~s}^{-1}$, and a REMPD time of $10 \mathrm{~s}$.

The transitions between spin states with maximal $\mathrm{J}$ and maximal magnitude of the projection $\mathrm{J}_{\mathrm{z}}= \pm \mathrm{J}$ (stretched states) of the levels $(\mathrm{v}, \mathrm{L})=(0,0)$ and $(\mathrm{v}, \mathrm{L})=(2,0)$ are not splitted by the magnetic field. They can be observed with arbitrary orientation of the magnetic field and are suitable for probing $\mathrm{THz}$ electric fields coupled off-resonantly to the rotational transition $(\mathrm{v}, \mathrm{L})=(0,0) \rightarrow(0,1)$ at $1.315 \mathrm{THz}$ or $(\mathrm{v}, \mathrm{L})=(2,0) \rightarrow(2,1)$ at $1.197 \mathrm{THz}$, respectively. The lightshifts, measured as the change of the two-photon frequency upon application of the THz-wave, are exploited to recover the magnitude of the $\mathrm{THz}$ electric field vector and its polarisation state. When the spectroscopy laser is referenced to a frequency standard, this approach allows absolute calibration of the amplitudes and the phases of the THz electric field components, using ab-initio calculations of $\mathrm{HD}^{+}$energy levels, their Zeeman shifts and dipole moments of the rotational transitions.

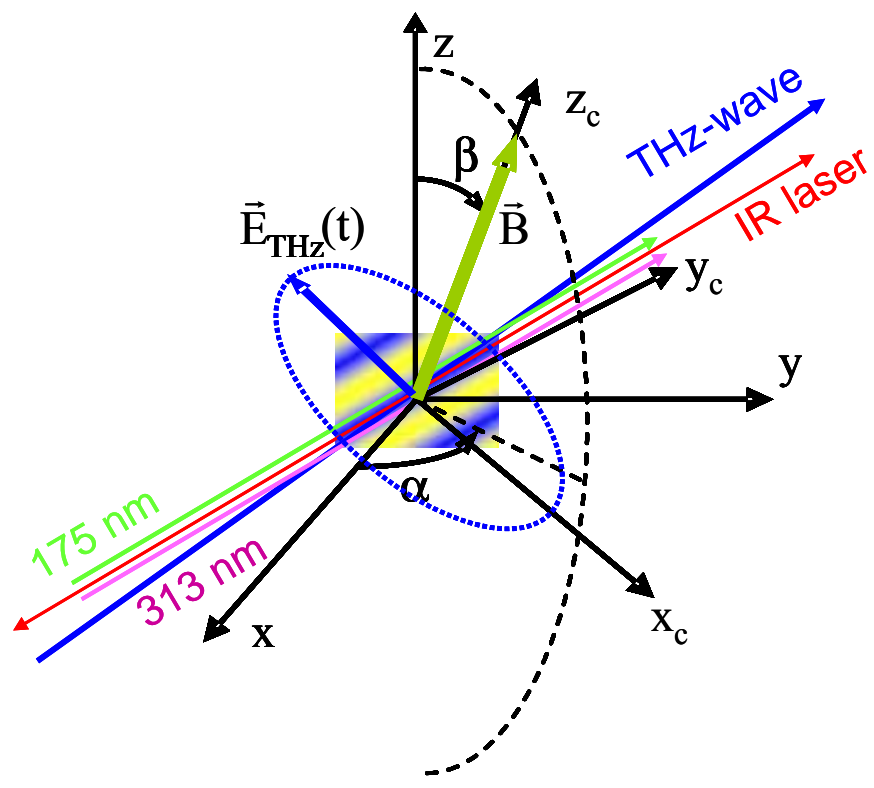

Figure 2. The experimental setup and reference frames. $\mathrm{HD}^{+}$ions embedded with laser cooled $\mathrm{Be}^{+}$ions in a Coulomb crystal are probed by two-photon spectroscopy. The magnetic field is generated with three pairs of coils oriented orthogonally that define the Laboratory Coordinate Frame $(\mathrm{x}, \mathrm{y}, \mathrm{z})$. The orientation of the magnetic field, defined with the Euler angles $(\alpha, \beta)$, is along the $\mathrm{z}_{\mathrm{c}}$ axis of the Cartesian Molecular Ion Coordinate frame $\left(\mathrm{x}_{\mathrm{c}}, \mathrm{y}_{\mathrm{c}}, \mathrm{z}_{\mathrm{c}}\right)$. Two couterpropagating waves from the IR laser are used for probing the $(\mathrm{v}, \mathrm{L})=(0,0) \rightarrow(2,0)$ and $(\mathrm{v}, \mathrm{L})=(0,0) \rightarrow(2,2)$ transitions of $\mathrm{HD}^{+}$. The $313 \mathrm{~nm}$ laser is used for cooling of $\mathrm{Be}^{+}$ions. The $175 \mathrm{~nm}$ laser is used for dissociation of the $(\mathrm{v}, \mathrm{L})=(2,0)$ level of $\mathrm{HD}^{+}$. The polarisation ellipse of the $\mathrm{THz}^{-}$ wave is retrieved from the measurements of the lightshifts of Zeeman components of the $(v, L)=(0,0) \rightarrow(2,0)$ transition.

The fractional frequency uncertainty is estimated with the expression of the Allan variance for the quantum projection noise limit :

$$
\sigma_{y}(\tau)=\frac{1}{\pi Q \sqrt{N_{\text {ion }}}} \times \sqrt{\frac{T_{c}}{\tau}}
$$

where $Q$, the quality factor of the two-photon transition, is expressed as $Q=f_{2 p h} / \Delta f_{H W H M}$ in terms of the half-linewidth, determined ultimately by the natural lifetimes of the $\mathrm{HD}^{+}$ion energy levels ${ }^{11}$. The cycle time $T_{c}$ is associated to a single measurement with $N_{\text {ion }}$ ions at two-photon resonance and successive measurements are averaged during an interrogation time $\tau$. Assuming the case of single ion spectroscopy experiment with $T_{c}=\tau$, the frequency uncertainty for $(\mathrm{v}, \mathrm{L})=(0,0) \rightarrow(2,0)$ line is estimated at $2.49 \mathrm{~Hz}$ and for $(\mathrm{v}, \mathrm{L})=(0,0) \rightarrow(2,2)$ line at $2.57 \mathrm{~Hz}$. 
The measurement of the Zeeman shift $\delta f$ of a two photon transition $\left|v L F S J, J_{Z}\right\rangle=\left|n_{1}, J_{Z 1}\right\rangle \rightarrow \mid n_{2}, J_{z 2}>$ allows to calculate the magnitude of the magnetic field using the relevant values for the theoretical parameters $\left\{U_{t h}\right\}=\left\{t_{n 1}, q_{n 1}, r_{n 1}, t_{n 2}, q_{n 2}, r_{n 2}\right\}$ of the Zeeman effect :

$$
\left\|\vec{B}\left(U_{t h}, \delta f\right)\right\|=\frac{-\left(t_{n 2} \cdot J_{z 2}-t_{n 1} \cdot J_{z 1}\right) \pm \sqrt{\left(t_{n 2} \cdot J_{z 2}-t_{n 1} \cdot J_{z 1}\right)^{2}+8 \delta f\left(r_{n 2} J_{z 2}^{2}-r_{n 1} J_{z 1}^{2}+q_{n 2}-q_{n 1}\right)}}{2\left(r_{n 2} J_{z 2}^{2}-r_{n 1} J_{z 1}^{2}+q_{n 2}-q_{n 1}\right)}
$$

When two different values are predicted for the magnitude of the magnetic field, the sign in the previous equation is selected such as the value of the magnetic field derived by Zeeman spectroscopy matches the value measured with an accurate magnetometer. The frequency uncertainty $\sigma_{\text {exp,2ph }}$ and the uncertainties of the parameters $\sigma_{\text {th,param }}=5 \times 10^{-4} \mathrm{kHz}$, assumed equal to one half of the last significant digit calculated in ref. ${ }^{4}$, contribute to the uncertainty of the magnetic field. The error propagation formula is exploited to calculate the total uncertainty of the magnetic field, by assuming that the theoretical parameters are correlated (correlation coefficients $\left.\operatorname{corr}\left(t_{n i}, q_{n i}\right)=\operatorname{corr}\left(q_{n i}, r_{n i}\right)=\operatorname{corr}\left(t_{n i}, r_{n i}\right)=1, \mathrm{i}=1,2\right)$.

The $(\mathrm{v}, \mathrm{L})=(0,0) \rightarrow(2,2)$ transition, with a transition probability by five times higher than that of the $(\mathrm{v}, \mathrm{L})=(0,0) \rightarrow(2,0)$ transition $^{12}$, has sensitive magnetic subcomponents. The measurement of the magnetic field may be performed by Zeeman spectroscopy of the subcomponent $\left|\mathrm{v}, \mathrm{L}, \mathrm{F}, \mathrm{S}, \mathrm{J}, \mathrm{J}_{2}>=\right| 0,0,1,2,2,-2>\rightarrow \mid 2,2,1,2,4,0>$, having the highest Zeeman shift of $719.655 \mathrm{kHz}$ at $B=10^{-4} \mathrm{~T}$. The magnitude of the magnetic field is determined with an uncertainty $<6.7 \times 10^{-10} \mathrm{~T}$. The dependence of the relative uncertainty with the magnitude of the magnetic field is plotted in Figure 3. The contribution of the theoretical uncertainties is approximatively four orders of magnitude smaller.

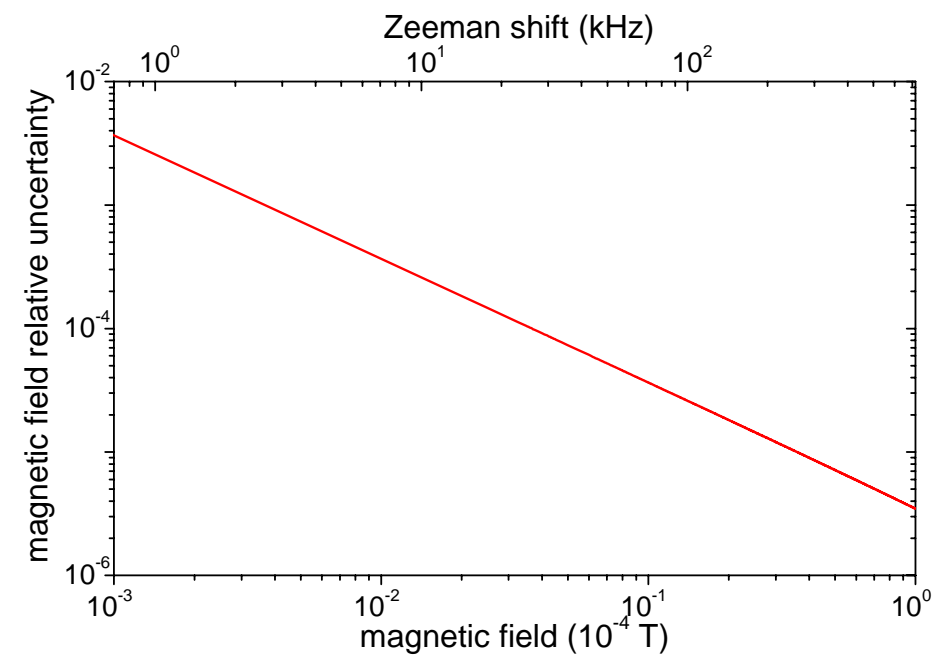

Figure 3. Relative uncertainty of the magnitude of the magnetic field determined by Zeeman spectroscopy of the $\left|\mathrm{v}, \mathrm{L}, \mathrm{F}, \mathrm{S}, \mathrm{J}, \mathrm{J}_{\mathrm{Z}}>=\right| 0,0,1,2,2,-2>\rightarrow \mid 2,2,1,2,4,0>$ transition of $\mathrm{HD}^{+}$. The frequency uncertainty $\sigma_{\text {exp }, 2 p h}=2.57 \mathrm{~Hz}$ is estimated with the quantum projection noise-limited instability for integration time $1 \mathrm{~s}$, cycle time $1 \mathrm{~s}$.

\section{LIGHTSHIFTS IN TWO-PHOTON SPECTROSCOPY OF HD ${ }^{+}$}

\subsection{Second-order $\mathrm{THz}$ electric field response of $\mathrm{HD}^{+}$energy levels}

Consider the interaction of a $\mathrm{HD}^{+}$ion with a classical $\mathrm{THz}$ electric field expressed as : 


$$
\overrightarrow{\mathrm{E}}(\mathrm{t})=\frac{1}{2} E_{L} \hat{\varepsilon} \times \mathrm{e}^{-i \omega t}+\text { c.c. }
$$

where $E_{L}$ is the complex amplitude of the THz-wave, $\hat{\varepsilon}$ is the complex polarization vector and $\omega$ is the angular frequency. The electric field vector is expressed in terms of the standard components $\hat{\varepsilon}_{q=\{-1,0,1\}}$ of the polarization vector:

$$
\begin{aligned}
E_{L} \hat{\varepsilon}=\sum_{q=\{-1,0,1\}}(-1)^{q} E_{L,-q} \hat{\varepsilon}_{q} \\
\text { with } \hat{\varepsilon}_{ \pm 1}=\mp \frac{\vec{e}_{x} \pm i \vec{e}_{y}}{\sqrt{2}}, \quad \hat{\varepsilon}_{0}=\vec{e}_{z}
\end{aligned}
$$

The coupling between the $\mathrm{HD}^{+}$energy levels and the $\mathrm{THz}$ electric field is expressed in the electric dipole approximation with the interaction Hamiltonian :

$$
\mathrm{V}=-\vec{d} \cdot \overrightarrow{\mathrm{E}}(\mathrm{t})=\frac{1}{2} E_{L}(\vec{d} \cdot \hat{\varepsilon}) \times \mathrm{e}^{-i \omega t}+H . c .
$$

where $\vec{d}$ is the electric dipole operator in the laboratory frame. When the THz electric field is far from the resonance with another energy level, the ac-Stark shift of an energy level, evaluated with the second-order perturbation theory ${ }^{13}$, reads:

$$
\delta E_{n}=-\frac{1}{4}\left|E_{L}\right|^{2} \sum_{r} \operatorname{Re}\left[\frac{\left\langle n\left|(\vec{d} \cdot \hat{\varepsilon})^{+}\right| r\right\rangle\langle r|(\vec{d} \cdot \hat{\varepsilon})| n\rangle}{E_{r}-E_{n}-\hbar \omega-i \hbar \frac{\gamma_{r}+\gamma_{n}}{2}}+\frac{\langle n \mid(\vec{d} \cdot \hat{\varepsilon}) r\rangle\left\langle r\left|(\vec{d} \cdot \hat{\varepsilon})^{+}\right| n\right\rangle}{E_{r}-E_{n}+\hbar \omega+i \hbar \frac{\gamma_{r}+\gamma_{n}}{2}}\right]
$$

in terms of the matrix elements of the dipole operator, the unperturbed energy levels $E_{r}, E_{n}$, and their decay rates $\gamma_{r}, \gamma_{n}$. The tensor formalism allows to express the matrix elements between Zeeman sublevels of the standard components $d_{-q}=\vec{d} \cdot \hat{\varepsilon}_{q}$ of the electric dipole operator :

$$
\begin{aligned}
& \left\langle v, L, F, S, J, J_{z}\left|d_{-q}\right| v^{\prime}, L^{\prime}, F^{\prime}, S^{\prime}, J^{\prime}, J_{z}^{\prime}\right\rangle=\mu_{v L, v^{\prime} L^{\prime}}(-1)^{J+J^{\prime}+S-J_{z}+1+L+L} \delta_{S, S^{\prime}} \\
& \times \sqrt{(2 J+1)\left(2 J^{\prime}+1\right)(2 L+1)\left(2 L^{\prime}+1\right)}\left(\begin{array}{ccc}
J & 1 & J^{\prime} \\
-J_{z} & q & J^{\prime}{ }_{z}
\end{array}\right)\left\{\begin{array}{ccc}
L^{\prime} & J^{\prime} & S \\
J & L & 1
\end{array}\right\}\left(\begin{array}{ccc}
L & 1 & L^{\prime} \\
0 & 0 & 0
\end{array}\right)
\end{aligned}
$$

in function of the reduced matrix elements $\mu_{v L, v^{\prime} L^{\prime}}$ of the dipole moment that have been calculated in the nonrelativistic approximation in ref. ${ }^{14}$.

\subsection{Dynamic polarizabilities of $\mathbf{H D}^{+}$energy levels}

Equation (7) can be expressed as the expectation value of a Stark-shift operator $\Theta(\omega)$ and further expanded in function of the squared magnitudes of the standard components of the $\mathrm{THz}$ electric field :

$$
\begin{gathered}
\delta E_{n}=\frac{\left|E_{L}\right|^{2}}{4}\left\langle v, L, F, S, J, J_{z}|\Theta(\omega)| v, L, F, S, J, J_{z}\right\rangle=-\frac{1}{4} \sum_{q=\{-1,0,1\}}(-1)^{q}\left|E_{L,-q}\right|^{2} \alpha_{n, q}(\omega) \\
\alpha_{n, q}(\omega)=\operatorname{Re}\left[\left\langle n\left|d_{-q} \cdot \frac{1}{\underline{\mathrm{H}}^{*}-\underline{\mathrm{E}}_{n}-\hbar \omega} \cdot d_{q}\right| n\right\rangle+\left\langle n\left|d_{-q} \cdot \frac{1}{\underline{\mathrm{H}}-\underline{\mathrm{E}}_{n}^{*}+\hbar \omega} \cdot d_{q}\right| n\right\rangle\right]
\end{gathered}
$$


by introducing the standard (labeled as $\sigma^{-}, \pi, \sigma^{+}$for $\left.\mathrm{q}=-1,0,1\right)$ dynamic polarizabilities of the $\mathrm{HD}^{+}$energy levels $\alpha_{n, q}(\omega)$, which are defined on the second line of eq. (9) using the complex operator $\underline{\mathrm{H}}=H+i \hbar \Gamma$, defined such as $\left\langle n|H| n^{\prime}\right\rangle=\delta_{n n^{\prime}} E_{n},\left\langle n|\Gamma| n^{\prime}\right\rangle=\delta_{n n^{\prime}} \gamma_{n} / 2$, and its eigenvalues $\underline{\mathrm{E}}_{n}=E_{n}+i \hbar \gamma_{n} / 2$.

In addition, the expression of the AC-Stark shift can be further developed by introducing the reduced scalar, vector and tensor $(K=0,1,2)$ polarizabilities for $\mathrm{HD}^{+}$ion hyperfine levels :

$$
\delta E_{n}=\frac{\left|E_{L}\right|^{2}}{4} \sum_{K=0,1,2}(-1)^{K} \sum_{\mu=-K \ldots K}(-1)^{\mu}\left\{\hat{\varepsilon}^{*} \otimes \hat{\varepsilon}\right\}_{K, \mu}(-1)^{J-J_{z}}\left(\begin{array}{ccc}
J & K & J \\
-J_{z} & -\mu & J_{z}
\end{array}\right) \alpha_{\nu L F S J}^{(K)}(\omega)
$$

in function of the irreducible tensor product components $\left\{\hat{\varepsilon}^{*} \otimes \hat{\varepsilon}\right\}_{K, \mu}$. The approach to calculate the reduced polarizabilities of the hyperfine $\mathrm{HD}^{+}$levels is indicated in the Appendix.

\subsection{Numerical calculation of the polarizabilities and their uncertainties}

The standard dynamic polarizabilities of the Zeeman subcomponents of the $(v, L)=(0,0)$ and $(v, L)=(2,0)$ states are calculated with eq. (9), by summing over all electric dipole couplings to sublevels of the $(v, L)=(0,1)$ and $(v, L)=(2,1)$ states, respectively. The standard dynamic polarizability :

$$
\alpha_{v, L=0, F, S, J, J_{z}, q}\left(f_{T H z}\right)=f\left(\left[\begin{array}{cc}
E_{r v}(v, L=0) & E_{r v}(v, L=1) \\
E_{h f}(v, L=0, F, S, J) & {\left[E_{h f}(v, L=1)\right]} \\
\Delta E_{z}(v, L=0, F, S, J ; B) & {\left[\Delta E_{z}(v, L=1 ; B)\right]}
\end{array}\right], \mu_{v 0, v 1}, \gamma_{(v, L)=(v, 0)}, \gamma_{(v, L)=(v, 1)} ; f_{T H z}\right)
$$

is a function of a set of theoretical parameters: the rovibrational, hyperfine and Zeeman energies, the dipole moment, the natural linewidths of energy levels (multiple values are regrouped within squared brackets), and two experimental parameters: the magnitude of the magnetic field and the polarization and the frequency of the THz-wave.

In order to avoid divergences and to maintain the approximation of far-detuned $\mathrm{THz}$ electric field, the contribution of the resonant coupling was neglected on a small frequency domain centered on each resonance $(10 \mathrm{~Hz}$ for the polarizabilities of the $(\mathrm{v}, \mathrm{L})=(0,0)$ states, $1.1 \mathrm{kHz}$ for the polarizabilities of the $(\mathrm{v}, \mathrm{L})=(2,0)$ states $)$.

The uncertainty of the dynamic polarizability is estimated conservatively with the root sum of squares of contributions from:

- the uncertainty of the theoretical rovibrational energies ${ }^{15}$, estimated with the assumed fractional value of $\sigma_{r}\left(E_{r v}\right)=10^{-12}$,

- the uncertainty of the theoretical hyperfine energies, assumed at one half of the last significant digit of the values from ref. ${ }^{9}$, that is $\sigma\left(E_{h f}\right)=500 \mathrm{~Hz}$,

- the uncertainty of the theoretical Zeeman energies calculated using eq. (1) with parameters given in ref. ${ }^{4}$, having accuracies of $\sigma\left(t_{n}\right)=\sigma\left(q_{n}\right)=\sigma\left(r_{n}\right)=0.5 \mathrm{~Hz}$,

- the uncertainty of the magnitude of the magnetic field, calculated with error propagation formula applied to eq. (3), by assuming an experimental frequency uncertainty of $\sigma_{\text {exp, } 2 p h}=2.49 \mathrm{~Hz}$, and correlated theoretical parameters from ref. ${ }^{4}$, with uncertainties of $\sigma_{\text {th,paramz }}=0.5 \mathrm{~Hz}$,

- the uncertainty of the theoretical dipole moments, assumed at one half of the last significant digit of the values calculated in ref. ${ }^{14}$, that is $\sigma\left(\mu_{00,01}\right)=\sigma\left(\mu_{20,21}\right)=1.3 \times 10^{-4}$ a.u., 
- the uncertainty of the theoretical radiative linewidths of the energy levels, estimated at $\sigma\left(\gamma_{(v, L)=(0,1)}\right)=2.5 \times 10^{-7} \mathrm{~Hz}$ and $\sigma\left(\gamma_{(v, L)=(2,0)}\right)=\sigma\left(\gamma_{(v, L)=(2,1)}\right)=0.49 \mathrm{~Hz}$ using values of energy level lifetimes from ref. ${ }^{11}$,

- $\quad$ the uncertainty of the THz-wave frequency, assumed at $10^{-12}$ in relative value.

First, the uncertainty of the Zeeman shift is calculated as the root sum of squares of the contributions of the uncertainties of the theoretical parameters and of the uncertainty of the magnitude of the magnetic field. Next, the uncertainty of the total energy of a Zeeman sublevel is calculated as the root sum of squares of the uncertainties of the rovibrational energy, the hyperfine energy and the Zeeman shift. The uncertainty of an electric dipole coupling term from the expression of the dynamic polarizability is calculated with the root sum of squares of the contributions from the total energy of each Zeeman sublevel, the dipole moment, the energy level linewidth and THz-wave frequency. Finally, the uncertainty of the dynamic polarizability is conservatively estimated as the root sum of squares of individual contributions of couplings between Zeeman sublevels.

At the limit of a static electric field, the value of the polarizability of a stretched state at zero magnetic field is $\alpha_{|0,0,1,2,2,2\rangle, 1}(\omega=0)=392.001$ a.u. , that agrees with the value calculated in ref. ${ }^{5}$. The fractional uncertainty is $2.9 \times 10^{-4}$.

Figure 4.(a) displays the standard dynamic polarizabilities of the stretched state $\mid 0,0,1,2,2,2>$ at a magnetic field $B=10^{-4}$ $\mathrm{T}$. The dependence for $\sigma^{+}$dynamic polarizability has a resonance corresponding to the coupling to the $\mid 0,1,1,2,3,3>$ level. The $\sigma^{-}$dynamic polarizability has two resonances in this frequency range, corresponding to the couplings to the $\mid 0,1,1,2,3,1>$ and $0,1,1,2,2,1>$ levels, respectively. The $\pi$ dynamic polarizability has two resonances corresponding to couplings to the $\mid 0,1,1,2,3,2>$ and $0,1,1,2,2,2>$ levels, respectively. The uncertainties are plotted in Figure 4(b). The increase of the magnitude of the dynamic polarizability, when the THz-wave is tuned near a resonance, is associated with an increase of the uncertainty.

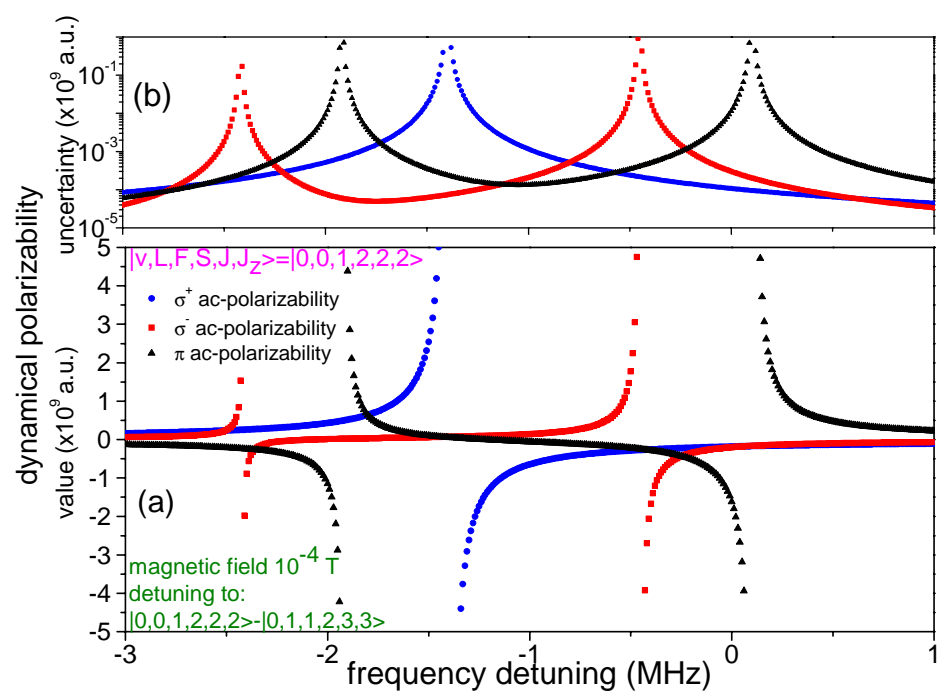

Figure 4. Dependence of the value and the uncertainty of the standard dynamic polarizability of the stretched state $\left|\mathrm{v}, \mathrm{L}, \mathrm{F}, \mathrm{S}, \mathrm{J}, \mathrm{J}_{\mathrm{Z}}>=\right| 0,0,1,2,2,2>$ on the frequency detuning to $1314945902.3 \mathrm{kHz}$ (corresponding to the hyperfine resonance $|\mathrm{v}, \mathrm{L}, \mathrm{F}, \mathrm{S}, \mathrm{J}>=| 0,0,1,2,2>\rightarrow \mid 0,1,1,2,3>$ ). Magnetic field $10^{-4} \mathrm{~T}$.

Each dipole-allowed transition between the Zeeman components of the $(\mathrm{v}, 0)$ and $(\mathrm{v}, 1)$ states constitutes a sensor for a $\mathrm{THz}$ electric field with a frequency tuned near the resonance frequency. The positions of the resonances can be adjusted by changing the magnitude of the magnetic field. Figure 5 indicates the dependence of the resonance frequencies on the 
magnitude of the magnetic field up to 5 Gauss for a set of hyperfine transitions detuned by $10 \mathrm{MHz}$ to the hyperfine-free rotational frequency. Each component has a dependence with a specific slope. The highest slope has an absolute value of about $7 \mathrm{GHz} / \mathrm{T}$.

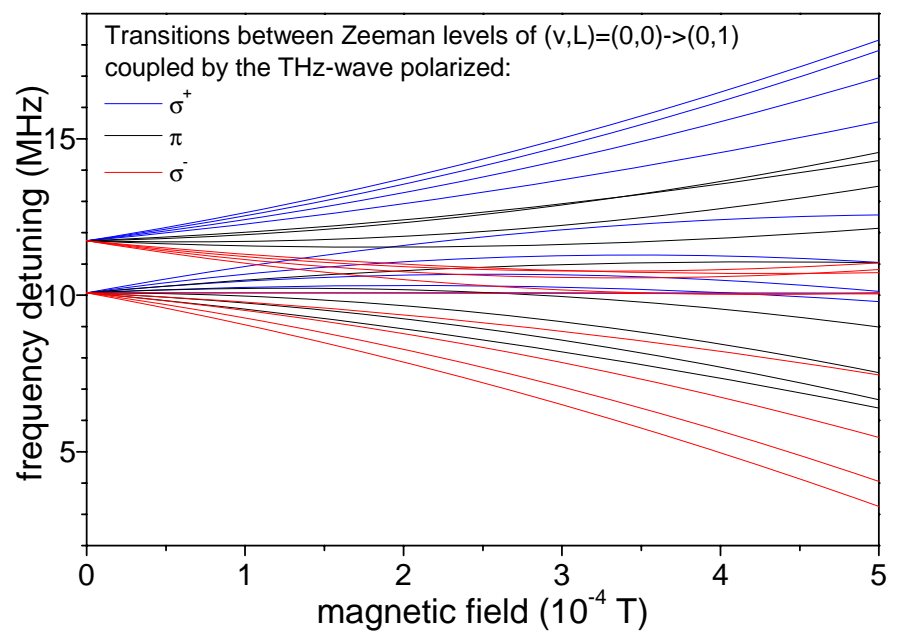

Figure 5. Magnetic field dependence of the resonance frequencies for selected Zeeman components of the rotational transition $|\mathrm{v}, \mathrm{L}>=| 0,0>\rightarrow \mid 0,1>$. The frequency detuning is calculated to $1314935827.3 \mathrm{kHz}$ (the hyperfine-free rotational frequency).

\subsection{Probing THz electric fields with lightshifts: accuracy and sensitivity issues}

Let's consider now the detection of a THz-wave with $\sigma^{+}$polarization tuned around $1.3 \mathrm{THz}$ from the measurement of the lightshift induced on the frequency of the two-photon transition between the stretches states. At a given value of the magnetic field, the lightshift can be translated to the THz-wave intensity by multiplication with the appropriate factor depending on the $\mathrm{THz}$ wave frequency and polarization. The dependence of the lightshift on the detuning of the THzwave is plotted in Figure 6.. For the given parameters of the THz-wave, the lightshift is higher than the uncertainty of the measurement of the two-photon transition for a detuning of a few $\mathrm{MHz}$ around the resonances with the relevant rotational transitions, that indicates the bandwidth of the THz-wave detection.

In order to evaluate the performances of precision and sensitivity in THz-wave detection by lightshift measurements, one should asses the uncertainties of the determination of THz-wave intensity. The frequency measurement errors translate to uncertainties of the lightshift and of the magnetic field magnitude. The uncertainties of the theoretical parameters contribute to the uncertainty of the dynamic polarizability. The overall contribution to the uncertainty of the THz-wave intensity is estimated with the error propagation formula:

$$
\sigma^{2}\left(I_{T H z}\right)=\frac{c_{F}^{2} \times \sigma^{2}(\delta f)+\left[I_{T H z} \partial \Delta \alpha\left(\left\{U_{t h}\right\}, q, B, f_{T H z}\right) / \partial B\right]^{2} \times \sigma^{2}(B)}{\left[\Delta \alpha\left(\left\{U_{t h}\right\}, q, B, f_{T H z}\right)\right]^{2}}
$$

in function of the lightshift measurement uncertainty $\sigma(\delta f)$ and the uncertainty of the magnetic field magnitude $\sigma(B)$. The prefactor $c_{F}=-\hbar c /\left(2 \pi a_{0}^{3}\right)$ is a combination of fundamental constants (the reduced Planck constant $\hbar$, the speed of light in vacuum $c$, the Bohr radius $a_{0}$ ). The ab-initio calculated differential dynamical polarizability $\Delta \alpha$ depends on a set of theoretical constants, the magnetic field, the polarization and the angular frequency of the THz-wave. Therefore, one can make a choice for different parameters, for example the Zeeman subcomponents of the two-photon transition, the 
magnitude of the magnetic field in order to minimize the uncertainty at particular intensity, polarization and frequency of the THz-wave.

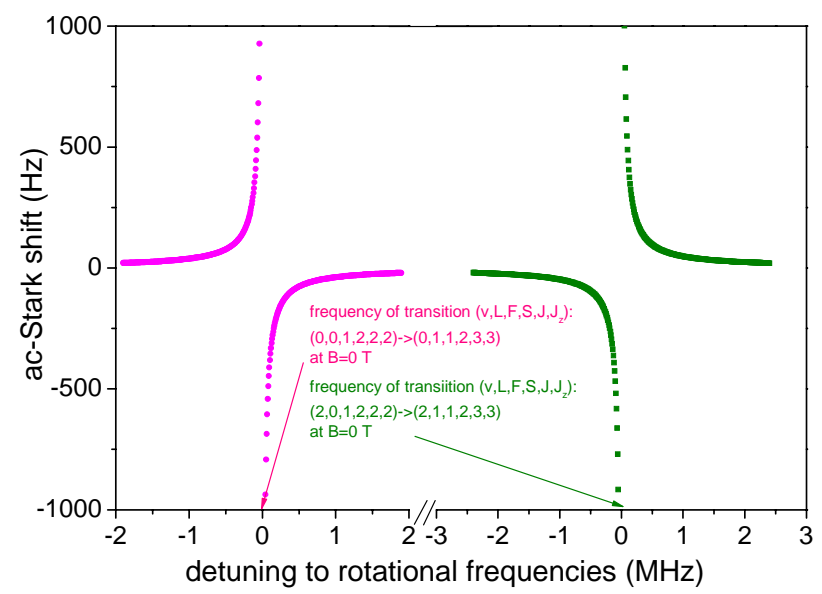

Figure 6. Dependence of the ac-Stark shift of the two-photon transition $\left|\mathrm{v}, \mathrm{L}, \mathrm{F}, \mathrm{S}, \mathrm{J}, \mathrm{J}_{\mathrm{Z}}>=\right| 0,0,1,2,2,2>\rightarrow \mid 2,0,1,2,2,2>$ on the frequency detuning to $1314945902.3 \mathrm{kHz}$ (resonance $|\mathrm{v}, \mathrm{L}, \mathrm{F}, \mathrm{S}, \mathrm{J}\rangle=|0,0,1,2,2>\rightarrow| 0,1,1,2,3>$ ), and $1196702588.7 \mathrm{kHz}$ (resonance $|\mathrm{v}, \mathrm{L}, \mathrm{F}, \mathrm{S}, \mathrm{J}\rangle=|2,0,1,2,2>\rightarrow| 2,1,1,2,3>$ ), respectively. Magnetic field $10^{-4} \mathrm{~T}$. THz-wave intensity $0.3 \mathrm{~W} / \mathrm{m}^{2}$, frequency $1314947502 \mathrm{kHz}$, polarization $\sigma^{+}$.
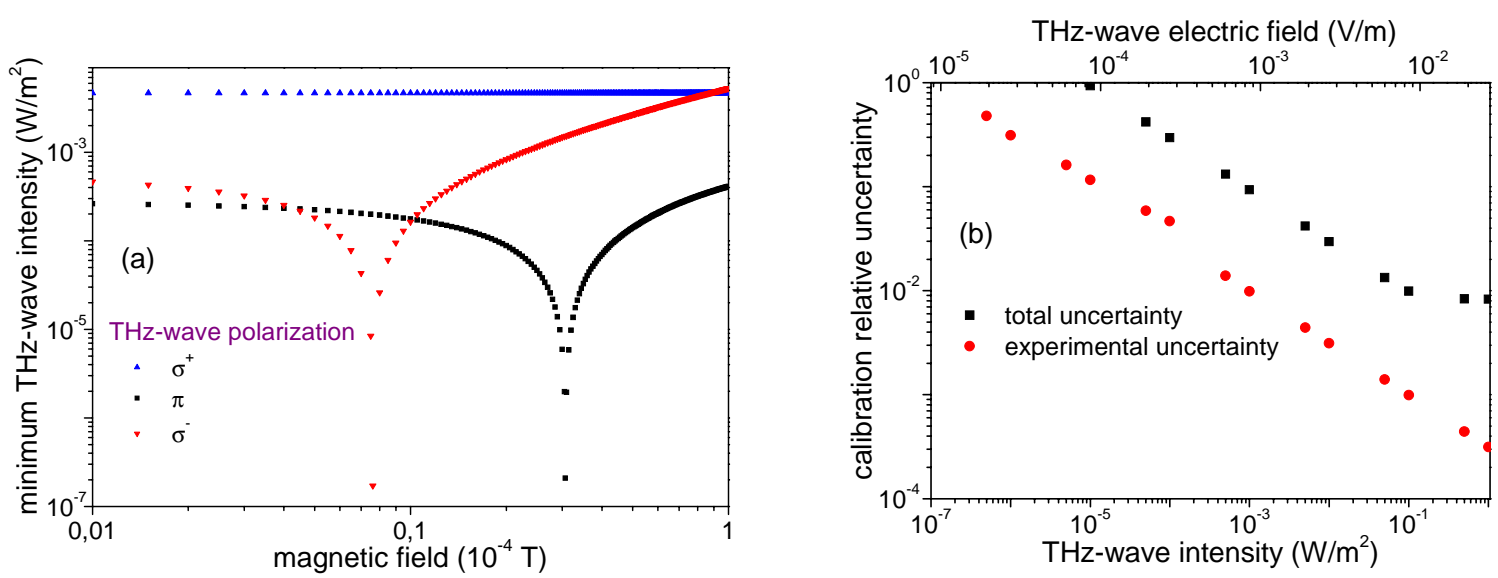

Figure 7. (a) Magnetic field dependence of the lowest THz-wave intensity that can be detected by frequency measurements of the lightshifts of the transition $\left|\mathrm{v}, \mathrm{L}, \mathrm{F}, \mathrm{S}, \mathrm{J}, \mathrm{J}_{\mathrm{Z}}>=\right| 0,0,1,2,2,2>\rightarrow \mid 2,0,1,2,2,2>$ at the quantum projection noise limit. THzwave frequency $1314947502.3 \mathrm{kHz}$ and polarizations $\sigma^{-}, \pi$ and $\sigma^{-+}$. (b) Dependence of the fractional uncertainty for THzwave calibration (calculation with combined experimental and theory errors in black squares, calculation for experimental errors only in red circles) with measurements of the lightshifts of the $\left|\mathrm{v}, \mathrm{L}, \mathrm{F}, \mathrm{S}, \mathrm{J}, \mathrm{J}_{\mathrm{Z}}>=\right| 0,0,1,2,2,2>\rightarrow \mid 2,0,1,2,2,2>$ two-photon transition. The magnetic field value is optimized to minimize total uncertainty. THz-wave frequency $1314947502.3 \mathrm{kHz}$ and polarization $\sigma^{-}$.

Figure 7.(a) displays the dependence of the sensitivity in function on the magnitude of the magnetic field, for the given THz-wave polarizations and frequency. The lowest THz-wave intensity is estimated with eq. (12) by taking into account solely the contribution coming from the frequency uncertainty $\sigma(\delta f)$. The limit is at $1.7 \times 10^{-7} \mathrm{~W} / \mathrm{m}^{2}$, which translates to a sensitivity to $\mathrm{THz}$ electric fields of $1.1 \times 10^{-5} \mathrm{~V} / \mathrm{m}$. 
Next, the uncertainty in the calibration of a THz-wave using lightshift measurements is estimated for an optimized value of the magnetic field in the range $0-10^{-4} \mathrm{~T}$, that minimizes the total uncertainty estimated with eq. (12). Figure 7.(b) displays the dependence of the fractional uncertainty of the THz-wave intensity. For THz-wave intensities higher than $0.1 \mathrm{~W} / \mathrm{m}^{2}$ the optimized value for the magnetic field is negative and therefore fixed at 0 . The best fractional uncertainty is at the $10^{-3}$ level. The fractional uncertainty is limited by the accuracy of the dynamic polarizability calculation at the $10^{-2}$ level, for a THz-wave intensity of $1 \mathrm{~W} / \mathrm{m}^{2}$. The contribution to the fractional uncertainty arising solely from the frequency measurement errors (this dependence is also plotted in Fig. 7.(b)) is more than an order of magnitude smaller than the total fractional uncertainty. The experimental fractional uncertainty is at the $10^{-4}$ level for a THz-wave intensity of $1 \mathrm{~W} / \mathrm{m}^{2}$.

\section{VECTOR TERAHERTZ ELECTROMETRY}

\subsection{Coordinate frames for $\mathbf{T H z}$-wave sensing}

The THz electric field vector is decomposed in three orthogonal linearly polarized components along the three axis of a fixed Cartesian laboratory coordinate frame $\operatorname{LCF}\left(\vec{e}_{x}, \vec{e}_{y}, \vec{e}_{z}\right)$ :

$$
\overrightarrow{\mathrm{E}}(\mathrm{t})=\sum_{j=\{x, y, z\}} \frac{E_{j} \vec{e}_{j}}{2} \times \mathrm{e}^{-i\left(\omega t+\varphi_{j}\right)}+\text { c.c. }
$$

The THz electric field is defined with real positive amplitudes $E_{j}$ and phases $\varphi_{j}$. One can fix, for simplicity, one phase to zero: $\varphi_{z}=0$. In this case, the $\mathrm{THz}$ electric field vector traces a polarization ellipse (PE) characterized with five parameters (three amplitudes and two relative phases). The magnetic field is generated with three coil pairs in Helmholtz configuration which are oriented orthogonally along $\vec{e}_{x}, \vec{e}_{y}, \vec{e}_{z}$, respectively. It is useful to define also the Cartesian molecular ion coordinate frame $\operatorname{MICF}\left(\vec{e}_{c, x}, \vec{e}_{c, y}, \vec{e}_{c, z}\right)$, such as the direction of $\vec{e}_{c, z}$ is along the direction of the magnetic field that defines the quantization axis. The $\mathrm{THz}$ electric field vector is expressed in the MICF using the standard components:

$$
\overrightarrow{\mathrm{E}}(\mathrm{t})=\sum_{q=\{-1,0,1\}}(-1)^{q} \frac{E_{-q} \vec{e}_{q}}{2} \times \mathrm{e}^{-i\left(\omega t+\varphi_{q}\right)}+\text { c.c. }
$$

with real positive amplitudes $E_{0}, E_{ \pm 1}$ and phases $\varphi_{0}, \varphi_{ \pm 1}$ having linear or circular polarization defined by $\vec{e}_{0}=\vec{e}_{c, z}, \vec{e}_{ \pm 1}=\mp\left(\vec{e}_{c, x} \pm i \vec{e}_{c, y}\right) / \sqrt{2}$. The orientation of $M I C F$ relative to $L C F$ can be changed by varying the currents in the coils. The relative orientation of the two coordinate frames is defined with the Euler angles $(\alpha, \beta, \zeta)$. Each standard component of the THz electric field, denoted with $E_{\pi, \sigma^{ \pm}}^{(\alpha, \beta)}$, couples off-resonantly to the $\pi$ or $\sigma^{ \pm}$Zeeman subcomponents of $(\mathrm{v}, \mathrm{L})=(\mathrm{v}, 0) \rightarrow(\mathrm{v}, 1)$ transitions and induces a lightshift in proportion with its squared amplitude.

The relations between electric field amplitudes in different coordinate system, can be calculated with the rotation matrix formalism, and $\operatorname{read}^{16}$ : 


$$
\begin{aligned}
\left(E_{\pi}^{(\alpha, \beta)}\right)^{2}= & E_{z}^{2} \cos ^{2} \beta \\
& +\left(E_{x}^{2} \cos ^{2} \alpha+E_{y}^{2} \sin ^{2} \alpha\right) \sin ^{2} \beta \\
& +E_{z} E_{x} \sin (2 \beta) \cos \alpha \cos \varphi_{x} \\
& +E_{z} E_{y} \sin (2 \beta) \sin \alpha \cos \varphi_{y} \\
& +E_{y} E_{x} \sin (2 \alpha) \sin ^{2} \beta \cos \left(\varphi_{x}-\varphi_{y}\right) \\
\left(E_{\sigma^{ \pm}}^{(\alpha, \beta)}\right)^{2}= & \frac{1}{2}\left(E_{x}^{2}+E_{y}^{2}+E_{z}^{2}-\left(E_{\pi}^{(\alpha, \beta)}\right)^{2}\right) \\
& \pm E_{x} E_{y} \cos \beta \sin \left(\varphi_{x}-\varphi_{y}\right) \\
& \mp E_{x} E_{z} \sin \alpha \sin \beta \sin \varphi_{x} \\
& \pm E_{y} E_{z} \cos \alpha \sin \beta \sin \varphi_{y}
\end{aligned}
$$

\subsection{Measuring the polarization ellipse of the THz-wave}

In the previous equations, the field amplitudes and phases in the laboratory frame $\left(E_{x}, E_{y}, E_{z}, \varphi_{x}, \varphi_{y}\right)$ are related to three standard components of the electric field in the molecular ion frame $E_{\pi}^{(\alpha, \beta)}, E_{\sigma^{+}}^{(\alpha, \beta)}$. For a given orientation of the magnetic field, three independent measurements of lightshifts $\delta f_{k}^{(\alpha, \beta)}, k=\{1,2,3\}$ allow to derive all standard components of the THz electric field, by solving a system of three equations with the appropriate differential standard dynamic polarizabilities. These measurements have to be independent, that is, the determinant of the system should be nonzero. That can be done by using different magnetic field magnitudes or probing on different two-photon transitions (for example, the Zeeman $\sigma^{ \pm}$ and $\pi$ components of a hyperfine transition or three different hyperfine transitions). In order to determine all parameters of the THz electric field in the laboratory frame, it is necessary also to change the orientation of the magnetic field. The choice of orientations of the magnetic field is linked to the periodicity of the trigonometric functions in eq. (15) and measurements for minimum two different orientations are required. Therefore, in order to determine five parameters of $\vec{E}(t)$ in the laboratory frame, five independent measurements are necessary for the standard components $E_{\pi}^{(\alpha, \beta)}, E_{\sigma^{ \pm}}^{(\alpha, \beta)}$ at two different orientations $(\alpha, \beta)$ of the magnetic field. For example, one can exploit the measurement of the set of amplitudes $\left(E_{\pi}^{(0,0)}, E_{\sigma^{+}}^{(0,0)}, E_{\sigma^{-}}^{(0,0)}, E_{\sigma^{+}}^{(0, \pi / 2)}, E_{\sigma^{-}}^{(0, \pi / 2)}\right)$ to derive analytically the parameters of the THz electric field in the laboratory frame ${ }^{16}$.

The experimental uncertainties of the THz electric field measurement arise from lightshift frequency measurements and from the uncertainties in setting the magnitude and the orientation of the magnetic field. Here, stray magnetic fields are neglected as well as nonorthogonality in relative orientation of the coil pairs. The magnitude of the magnetic field generated along $\vec{e}_{x}, \vec{e}_{y}, \vec{e}_{z}$, respectively, using separately each pair of coils can be calibrated with Zeeman spectroscopy, as discussed previously. In addition, errors in the calibration of the $\mathrm{THz}$ electric field arise from the uncertainties of the standard dynamic polarizabilities.

Let's consider detection of a THz electric field oscillating at frequency $f_{T H z}=1314947502.3 \mathrm{kHz}$ with the following parameters in the laboratory frame $\left(E_{x}=E_{y}=E_{z}=15.83 \mathrm{mV} / \mathrm{m}, \varphi_{x}=\pi / 4, \varphi_{y}=\pi / 3\right)$. A set of six theoretical lighshifts are calculated for the two-photon transition between the stretched states $\left|\mathrm{V}, \mathrm{L}, \mathrm{F}, \mathrm{S}, \mathrm{J}, \mathrm{J}_{\mathrm{Z}}>=\right| 0,0,1,2,2,2>\rightarrow \mid 2,0,1,2,2,2>$, for two orientations of the magnetic field $(\alpha, \beta)=(0,0),(\alpha, \beta)=(0, \pi / 2)$, and three different values of the magnitude of the magnetic field $B_{1}=10^{-6} \mathrm{~T}, B_{2}=5 \times 10^{-6} \mathrm{~T}, B_{3}=10^{-5} \mathrm{~T}$. The experimental lightshifts are simulated by adding to the theoretical lightshifts random values with a Gaussian distribution with zero mean and covariance equal to the squared uncertainty of the frequency measurements at the quantum projection noise $\operatorname{limit} \delta f_{n, \exp }^{(\alpha, \beta)}=\delta f_{n, \text { theor }}^{(\alpha, \beta)}+\boldsymbol{\aleph}\left(0, \sigma_{e x p, 2 p h}^{2}\right)$. The standard components of the $\mathrm{THz}$ electric field are subsequently derived using the differential standard dynamic polarizabilities, estimated as the theoretical values plus a random contribution with a Gaussian distribution with zero mean and variance equal to the squared uncertainty of theoretical calculation $\Delta \alpha_{n, q}\left(f_{T H z}, B\right)=\Delta \alpha_{n, q, \text { theor }}\left(f_{T H z}, B\right)+\aleph\left(0, \sigma_{\Delta \alpha_{n, q, \text { theor }}\left(f_{T H z}, B\right)}\right)$. From the calculated values of the standard 
components, all parameters of the $\mathrm{THz}$ electric field in the laboratory frame are determined analytically. The polarization ellipse of the THz electric field is represented on Figure 8 for 10 simulated sets of lightshifts.

The values and the uncertainties of the parameters of the THz-wave determined from 100 simulations of lightshifts are:

$$
\begin{aligned}
E_{x} & =15.88(92) \mathrm{mV} / \mathrm{m} \\
E_{y} & =15.74(72) \mathrm{mV} / \mathrm{m} \\
E_{z} & =15.8313(30) \mathrm{mV} / \mathrm{m} \\
\varphi_{x} & =0.781(60) \\
\varphi_{y} & =1.047(10)
\end{aligned}
$$

The accuracy of the calibration is better than $5 \%$ for the components of the electric field and better than $10 \%$ for the phases. The shift between the initial assumed value and the determined value for each parameter of the THz-wave is consistent with the calculated uncertainty. Precision measurements of lightshifts for different orientations of the magnetic field allow full characterization the $\mathrm{THz}$ electric field in the laboratory frame and, therefore, represent feasible alternatives to the Rabi-rate measurements with Rydberg atoms ${ }^{16,17}$.

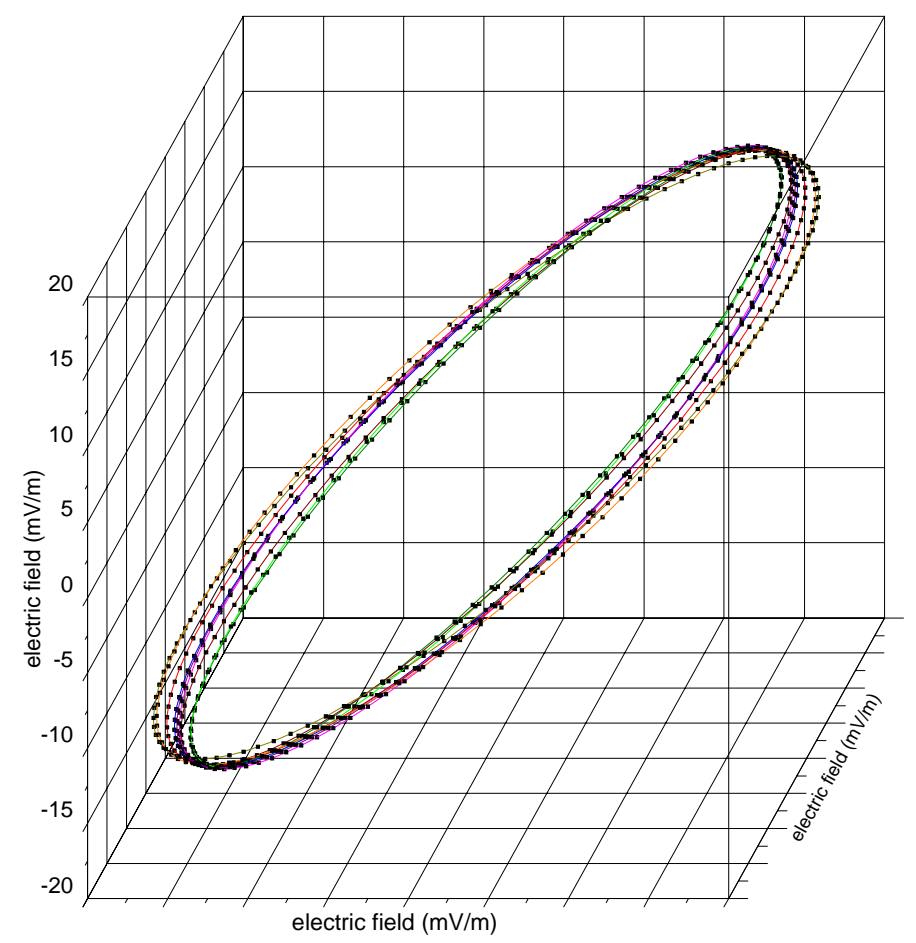

Figure 8. Determination of the polarization ellipse of the electric field of a THz-wave at $1314945902.3 \mathrm{kHz}$ from frequency measurements of the lightshifts of the two-photon transition $\left|\mathrm{v}, \mathrm{L}, \mathrm{F}, \mathrm{S}, \mathrm{J}, \mathrm{J}_{Z}>=\right| 0,0,1,2,2,2>\rightarrow \mid 2,0,1,2,2,2>$.

\section{CONCLUSION}

This contribution demonstrates that the measurements of the frequency shifts induced by external fields on narrow twophoton transitions of cold trapped $\mathrm{HD}^{+}$ions are feasible approaches for measuring electromagnetic fields. The Zeeman shift for a selected hyperfine component of a two-photon rovibrational transition is exploited for determination of the magnitude of a static magnetic field with an uncertainty at the $10^{-10} \mathrm{~T}$ level. The magnitude of the electric field of a THzwave is determined by the lightshift that is induced on a selected Zeeeman sub-component of a two-photon transition. A THz-wave with an intensity of $1 \mathrm{~W} / \mathrm{m}^{2}$ can be calibrated with an accuracy limited at the $10^{-2}$ level by the accuracy of the theoretical calculations and at the $10^{-4}$ level by the experimental errors. In addition, a self-consistent numerical approach 
for retrieval of the full polarisation ellipse is demonstrated for a selected THz-wave. The calibration accuracy is better than $5 \%$ for the electric field components and better than $10 \%$ for the phases..

\section{APPENDIX}

The reduced polarizability is expressed as :

$$
\alpha_{n J}^{(K)}(\omega)=(-1)^{K+2 J} \sqrt{2 K+1} \sum_{n^{\prime} J^{\prime}}\left[\begin{array}{l}
\left\{\begin{array}{ccc}
1 & K & 1 \\
J & J^{\prime} & J
\end{array}\right\}\left\langle n J\|d\| n^{\prime} J^{\prime}\right\rangle\left\langle n^{\prime} J^{\prime}\|d\| n J\right\rangle \\
\left.\times R e\left(\begin{array}{c}
\frac{1}{E_{n J}-E_{n^{\prime} J^{\prime}}+\hbar \omega+i \hbar\left(\gamma_{n J}+\gamma_{n^{\prime} J^{\prime}}\right) / 2} \\
+\frac{(-1)^{K}}{E_{n J}-E_{n^{\prime} J^{\prime}}-\hbar \omega-i \hbar\left(\gamma_{n J}+\gamma_{n^{\prime} J^{\prime}}\right) / 2}
\end{array}\right]\right)
\end{array}\right]
$$

where $n=(v, L, F, S), n^{\prime}=\left(v^{\prime}, L^{\prime}, F^{\prime}, S^{\prime}\right)$ are the quantum numbers for the relevant energy levels and $\left\langle n^{\prime} J^{\prime}\|d\| n J\right\rangle$ are the reduced matrix elements of the dipole operator. In the case where the detunings to the intermediary energy levels are larger than the hyperfine structure splittings, the reduced polarizability can be further expanded:

$$
\alpha_{n J}^{(K)}(\omega)=(-1)^{L+S+J+K}(2 J+1)\left\{\begin{array}{ccc}
J & K & J \\
L & S & L
\end{array}\right\} \alpha_{n L}^{(K)}(\omega)
$$

with the reduced polarizability for a rovibrational level :

$$
\alpha_{v L}^{(K)}(\omega)=(-1)^{K+2 L+1} \sqrt{2 K+1} \sum_{v^{\prime} L^{\prime}}\left[\begin{array}{l}
\left\{\begin{array}{lll}
1 & K & 1 \\
L & L^{\prime} & L
\end{array}\right\}\left\langle n L\|d\| n^{\prime} L^{\prime}\right\rangle\left\langle n^{\prime} L^{\prime}\|d\| n L\right\rangle \\
\times R e\left(\begin{array}{l}
\frac{1}{E_{n^{\prime} J^{\prime}}-E_{n J}-\hbar \omega-i \hbar\left(\gamma_{n J}+\gamma_{n^{\prime} J^{\prime}}\right) / 2} \\
+\frac{(-1)^{K}}{E_{n^{\prime} J^{\prime}}-E_{n J}+\hbar \omega+i \hbar\left(\gamma_{n J}+\gamma_{n^{\prime} J^{\prime}}\right) / 2}
\end{array}\right)
\end{array}\right]
$$

and the reduced matrix element of the dipole operator :

$$
\left\langle n L\|d\| n^{\prime} L^{\prime}\right\rangle=\mu_{v L, v^{\prime} L^{\prime}}(-1)^{L^{\prime}} \sqrt{(2 L+1)\left(2 L^{\prime}+1\right)}\left(\begin{array}{ccc}
L & 1 & L^{\prime} \\
0 & 0 & 0
\end{array}\right)
$$

\section{REFERENCES}

[1] Fan, H., et al, “Atom based RF electric field sensing,” J. Phys. B 48 (20), 202001 (2015).

[2] Sedlacek, J., et al, "Microwave electrometry with Rydberg atoms in a vapour cell using bright atomic resonances," Nat. Phys. 8 (11), 819-824 (2012).

[3] Korobov, V.I., Hilico, L. and Karr, J.-P., "Fundamental transitions and ionization energies of the hydrogen molecular ions with few ppt uncertainty," Phys. Rev. Lett. 118 (23), 233001 (2017).

[4] Bakalov, D., Korobov, V.I. and Schiller, S., "Magnetic field effects in the transitions of the HD" molecular ion and precision spectroscopy," J. Phys. B 44 (2), 025003 (2011).

[5] Schiller, S., Bakalov, D., Bekbaev, A.K. and Korobov, V.I., "Static and dynamic polarizability and the Stark and blackbody-radiation frequency shifts of the molecular hydrogen ions $\mathrm{H}_{2}^{+}, \mathrm{HD}^{+}$, and $\mathrm{D}_{2}{ }^{+}$, " Phys. Rev. A 89 (5), 052521 (2014). 
[6] Biesheuvel, J., Karr, J.-P., Hilico, L., Eikema, K.S.E., Ubachs, W. and Koelemeij, J.C.J., "Probing QED and fundamental constants through laser spectroscopy of vibrational transitions in $\mathrm{HD}^{+}$," Nat. Commun. 7, 10385 (2016).

[7] Alighanbari, S., Hansen, M.G., Korobov, V.I. and Schiller, S., "Rotational spectroscopy of cold, trapped molecular ions in the Lamb-Dicke regime," Nat. Phys. 14 (6), 555-559 (2018).

[8] Constantin, F.L., "Double-resonance two-photon spectroscopy of hydrogen molecular ions for improved determination of fundamental constants," IEEE Trans. Instrum. Meas. 68 (6), 2151-2159 (2019).

[9] Bakalov, D., Korobov, V.I. and Schiller, S., "High-precision calculation of the hyperfine structure of the HD" ion," Phys. Rev. Lett. 97 (24), 243001 (2006).

[10] Roth, B., Blythe, P., Wenz, H., Daerr, H. and Schiller, S., "Ion-neutral chemical reactions between ultracold localized ions and neutral molecules with single-particle resolution," Phys. Rev. A 73 (4), 042712 (2006).

[11] Amitay, Z., Zajfman, D. and Forck, P., "Rotational and vibrational lifetime of isotopically asymmetrized homonuclear diatomic molecular ions," Phys. Rev. A 50 (3), 2304-2308 (1994).

[12] Karr, J.-P., Kilic, C., Hilico, L., "Energy levels and two-photon transition probabilities in the HD ${ }^{+}$ion,” J. Phys. B 38 (7), 853-866 (2005).

[13]Le Kien, F., Schneeweiss, P. and Rauschenbeutel, A., "Dynamical polarizability of atoms in arbitrary light fields: general theory and application to cesium," Eur. Phys. J. D 67 (5), 92 (2013).

[14] Bakalov, D. and Schiller S., "Static Stark effect in the molecular ion HD"," Hyperfine Interact. 210 (1-3), 25-31 (2012).

[15] Moss, R.E., “Calculations for vibration-rotation levels of $\mathrm{HD}^{+}$, in particular for high N,” Mol. Phys. 78 (2), 371 405 (1993).

[16] Koepsell, J., Thiele, T., Deiglmayr, J, Wallraff, A. and Merkt, F., "Measuring the polarisation of electromagnetic fields using Rabi-rate measurements with spatial Resolution : Experiment and theory," Phys. Rev. A 95 (5), 053860 (2017).

[17] Thiele, T., Lin, Y., Brown, M.O. and Regal, C.A., "Self-calibrating vector atomic magnetometry through microwave polarization reconstruction," Phys. Rev. Lett 121 (10), 153202 (2018). 